\title{
Inhibiteurs \\ de mTOR \\ Des antiprolifératifs \\ pléiotropiques
}

> L'étude des propriétés cellulaires de la rapa-

Nicolas Pallet, Philippe Beaune, Eric Thervet,

mycine (Sirolimus, Rapamune ${ }^{\circledR}$ ) a permis la

Christophe Legendre, Dany Anglicheau

découverte chez la levure puis chez les mammifères des fonctions de la protéine kinase TOR (target of rapamycin). Chez les mammifères, mTOR (mammalian target of rapamycin) est un contrôleur central de la croissance cellulaire en réponse aux facteurs de croissance et aux nutriments. L'activation de mTOR a de nombreuses cibles et concerne notamment la régulation de la machinerie traductionnelle. Cette régulation fait intervenir deux effecteurs : l'activation de la kinase S6Kl et la phosphorylation de 4E-BPl. La rapamycine et ses analogues, le temsirolimus et l'évérolimus, en inhibant mTOR, inhibent la croissance de nombreux types cellulaires, expliquant ainsi les différentes voies de développement en clinique. En transplantation d'organes solides, la rapamycine a prouvé son efficacité dans la prévention du rejet aigu d'allogreffe. En oncologie, la rapamycine et les molécules apparentées exercent un effet antitumoral in vitro et in vivo sur un très grand nombre de types cellulaires. $\varepsilon n$ pathologie coronarienne, les endoprothèses enrobées de rapamycine ont conduit à une réduction significative de la resténose intrastent. Enfin, la rapamycine est en cours d'essai dans les phacomatoses et la polykystose rénale autosomique dominante, maladies au cours desquelles les anomalies de croissance cellulaire tiennent un rôle central. <

\section{Les protéines kinases TOR sont les cibles de la rapamycine}

La rapamycine (Rapa), découverte il y a trente ans dans le sol de l'île de Pâques, est un macrolide lipophile synthétisé par le champignon Streptomyces hygroscopicus. L'étude de ses mécanismes d'action a permis de comprendre le rôle central des complexes TOR dans le

contrôle de la croissance cellulaire.

La première étape a été la découverte de la liaison de la Rapa à l'immunophilline FK-BP12 (FK-binding protein 12) qui est un complexe inhibiteur de la croissance de Saccharomyces cerevisiæ. La mise en évidence d'une résistance aux effets antiprolifératifs du complexe FK-BP12/ Rapa chez des levures dont les gènes TORI et TOR2 étaient mutés a permis d'identifier les protéines TOR comme des cibles potentielles de la Rapa. Ces mutations, qui concernent le domaine FRB (FKBP-rapamycin binding domain) localisé en aminoterminal de TOR, à proximité du site catalytique de la kinase, empêchent le complexe FKBP12/Rapa de se lier à TOR et de l'inhiber [1, 2].

Les levures portent deux gènes homologues, TORI et TOR2, alors que les mammifères ne sont porteurs que

Article reçu le 26 janvier 2006, accepté le 17 mai 2006. 
d'un seul gène TOR. Les protéines TOR (280 kDa) sont apparentées à la famille des PIKK (phosphatidylinositol kinase-related kinases) et sont structurellement et fonctionnellement analogues à la protéine mTOR découverte, par la suite, chez les mammifères. Le complexe Rapa/FKBP-12 serait responsable, en se fixant au FRB, d'un encombrement stérique empêchant mTOR de former un complexe multiprotéique fonctionnel [3].

\section{Les complexes TOR contrôlent la croissance cellulaire}

La croissance cellulaire (augmentation de la masse cellulaire) et la prolifération cellulaire (augmentation du nombre de cellules) sont deux mécanismes distincts mais nécessairement couplés dans la constitution de tissus sains ou tumoraux.

Les fonctions des protéines TORl et TOR2 sont remplies chez la levure par deux complexes protéiques, TORCl et TORC2, respectivement. Le complexe TORCl contrôle la masse cellulaire en régulant la biosynthèse des ribosomes par activation du facteur transcriptionnel FHLl, en contrôlant le transport membranaire d'acides aminés par l'intermédiaire des protéines membranaires TAT2 et GAP1, et en régulant l'autophagie, l'activité du facteur transcriptionnel STAT3 et les voies de signalisation impliquant la protéine kinase C [4].

Le complexe TORC2, et son homologue mammifère mTORC2, sont responsables du contrôle spatial de la croissance cellulaire par l'intermédiaire de la voie de signalisation dépendante des RHO GTPases qui contrôle la formation du cytosquelette d'actine. mTORC2 est constitué de rictor (mAV03), mLST8 (G $\beta \mathrm{L}$ ) et de mTOR [5].

Chez les mammifères, I'homologue de TORCl, mTORCl, est constitué de la protéine raptor (regulatory associated protein of $m$ TOR), de mLST8 (G $\beta \mathrm{L}$ ) et de mTOR $\beta$. Seul mTORCl est inhibé par la Rapa. mTORCl est impliqué dans la régulation traductionnelle via la $P 70^{56 \mathrm{~K}}(\mathrm{~S} 6 \mathrm{Kl})$ et $4 \varepsilon-\mathrm{BPl}$ (4E-binding protein 1 ) et dans la biogenèse des ribosomes en contrôlant la RNA polymérase I (Pol I) via le facteur transcriptionnel RRN3/TIFlA (Figure 1). L'inhibition de mTOR par la Rapa induit une baisse de $15 \%$ à $20 \%$ de l'activité traductionnelle, contribuant ainsi

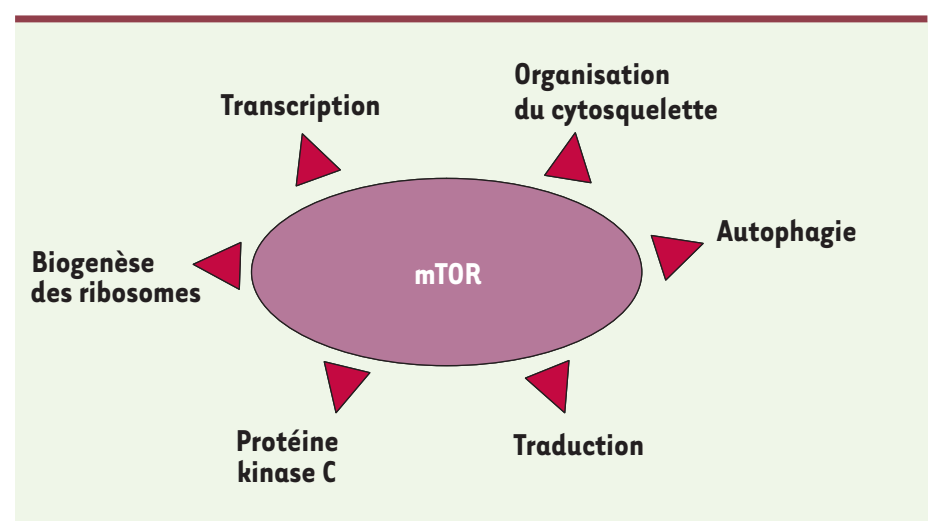

Figure 1. Fonctions cellulaires régulées par mTOR. mTOR contrôle une grande variété de fonctions cellulaires impliquées dans la croissance et la prolifération cellulaire. à une réduction de la croissance cellulaire. Les autres fonctions de mTOR dans le contrôle de la croissance cellulaire sont la régulation des apports cellulaires en nutriments et la transcription de gènes impliqués dans certaines voies métaboliques [6] (Figure 2).

Les fonctions de mTOR indépendantes de la Rapa sont probablement sous estimées, comme en témoigne le phénotype des souris $m T O R^{-}{ }^{-}$qui est plus sévère que celui des embryons de souris traités par rapamycine [7].

\section{Place de mTOR dans le contrôle de la traduction}

Le contrôle de l'activité traductionnelle est la fonction de mTOR qui a été initialement découverte et qui est la mieux décrite (Figure 3).

mTORCl est responsable de la phosphorylation et de l'activation de la protéine kinase $56 \mathrm{Kl}$ qui, à son tour, phosphoryle la protéine ribosomale S6. Il était initialement considéré que cette voie de signalisation conduisait à la traduction d'ARNm comportant une série de motifs pyrimidines, les 5'TOP (terminal oligopyrimidine tract). Ces ARNm codent des composants de l'appareil traductionnel comme des protéines ribosomales et des facteurs d'élongation. Des données récentes contestent ce modèle et les mécanismes par lesquels mTORCl contrôle la traduction des 5'TOP ARN restent à démontrer [8].

mTOR phosphoryle également $4 \varepsilon-B P 1$ qui est un inhibiteur de la traduction. Lorsque $4 \varepsilon-B P 1$ est phosphorylée, elle se dissocie du facteur traductionnel elF4E (elongation initiator factor $4 \varepsilon$ ) qui peut alors se lier à la coiffe 7-méthyl-guanosine localisée en 5' de certains ARNm (cycline D3, c-myc ou VEGF, vascular endothelial growth factor) et former un complexe initiateur de leur traduction. Ce complexe, appelé elF4F, comporte l'hélicase eIF4A et la protéine de structure eIF4G, et facilite la liaison de la sous unité ribosomale $40 S$ à l'ARNm. L'inhibition de mTOR empêche donc la phosphorylation de $4 \varepsilon-B P l$ et, par conséquent, la libération du facteur traductionnel elF4E [9].

\section{Voies de régulation de TOR}

La principale voie de signalisation activant mTOR implique l'axe IRS (insulin receptor substrate)/PI3 kinase (Figure 2). L'activation des récepteurs par l'insuline ou I'IGF (insulin growth factor) active I'IRS, recrute les PI3 kinases qui transforment les phosphatidylinositol-4,5,phosphates en phosphatidylinositol-3,4,5,-phosphates. Cette transformation est inhibée par le suppresseur de tumeur PTEN (phosphatase and tensin homolog deleted on chromosome 10). En aval de la PI3K, les voies de 
contrôle de la croissance cellulaire impliquent PDKl (phosphoinositide-dependent protein kinase 1) qui active Akt par phosphorylation [10]. Akt inhibe la tubérine par phosphorylation.

mTOR est régulée par l'hamartine et la tubérine, codées par les gènes TSCl et TSC2. TSCl et 2 sont des suppresseurs de tumeurs qui forment un hétérodimère inhibant la protéine Rheb (ras homolog enriched in brain), laquelle active normalement mTOR [11]. Il a été montré que le VEGF et l'interféron $\gamma$ activaient également cette voie de signalisation. Certains acides aminés comme la leucine et l'arginine activeraient mTORCl directement mais les mécanismes exacts sont mal compris.

\section{Utilisation clinique des inhibiteurs de mTOR}

\section{Inhibition de mTOR \\ en transplantation d'organes solides}

La Rapa est un puissant inhibiteur de l'activation des lymphocytes $T$ qui restent bloqués en phase $\mathrm{Gl}$ tardive après stimulation allogénique. Cet effet $a$ conduit à son utilisation dans le cadre de la prévention du rejet aigu d'allogreffe. La Rapa est apparue initialement comme une molécule très prometteuse devant l'absence apparente de néphrotoxicité et a été développée comme substitution aux inhibiteurs de la calcineurine en raison de leurs effets secondaires. Son autorisation de mise sur le marché en Europe a été délivrée en 2001 . Kahan et al. [12] ont montré, dans une étude prospective randomisée, que l'incidence des rejets aigus était inférieure, dans les groupes recevant de la Rapa à $2 \mathrm{mg}$ et $5 \mathrm{mg}$ en association avec la ciclosporine ( $\operatorname{Cs} A$ ), à celle des patients recevant I'association CsA/azathioprine (AZA) $(16,8 \%, 12 \%$ versus $32,3 \%$, respectivement). Cependant, la clairance de créatinine à trois mois était de $62 \mathrm{ml} / \mathrm{min}(p<0,01$ versus $A Z A), 59 \mathrm{ml} / \mathrm{min}$ ( $p<0,001$ versus AZA) et $69 \mathrm{ml} / \mathrm{min}$ dans les groupes recevant respectivement la Rapa à $2 \mathrm{mg}$, à $5 \mathrm{mg}$ ou l'AZA et, un an après la transplantation, la clairance de la créatinine était de $62 \mathrm{ml} / \mathrm{min}$ ( $p<0,05$ versus AZA), $55 \mathrm{ml} / \mathrm{min}(p<0,001$ versus AZA), et $68 \mathrm{ml} /$ min, respectivement. Cette étude prospective a ainsi démontré l'efficacité de la Rapa en association avec la CsA mais a également été la première à montrer l'effet délétère de cette association sur la fonction rénale. Ces résultats ont conduit la FDA à recommander d'interrompre la CsA trois mois après la transplantation en cas d'association initiale avec la Rapa.

Plusieurs études récentes ont suggéré un effet bénéfique de l'inhibition de mTOR en transplantation pulmonaire, indépendamment de l'effet immunosuppresseur, mais directement lié aux conséquences antiprolifératives sur les lésions de bronchiolite oblitérante du greffon pulmonaire [13].

La capacité des inhibiteurs de mTOR à inhiber in vitro la croissance des cellules musculaires lisses a été la raison de leur évaluation en cas de coronaropathie du greffon cardiaque, caractérisée histologiquement par une prolifération de ces cellules. Une étude randomisée a évalué l'efficacité et la tolérance de l'évérolimus, un dérivé de la rapamycine, en comparaison de celles de l'AZA chez les transplantés cardiaques récents. Cette étude a montré l'intérêt de l'évérolimus pour la prévention des rejets aigus et la survie des patients par rapport à I'AZA, mais a aussi révélé que les paramètres de remaniement de la paroi coronarienne étaient significativement moins sévères chez les patients traités par évérolimus. [14] (Tableau I).

\begin{tabular}{|c|c|c|}
\hline Type cellulaire & Application clinique & Références \\
\hline Lymphocytes T & Immunossuppression & [12] \\
\hline \multirow{2}{*}{ Myofibroblastes } & Stents enrobés & {$[15]$} \\
\hline & Coronaropathie de greffon cardiaque & [14] \\
\hline Fibroblastes & Bronchiolite oblitérante du greffon pulmonaire & [13] \\
\hline Cellules tubulaires rénales & Polykystose rénale autosomique dominante & \\
\hline $\begin{array}{l}\text { Cancers du sein, cancer du rein, glioblastome, cancer bronchique } \\
\text { non à petites cellules, mélanome, astrocytome anaplasique, } \\
\text { mésothélome, sarcome des tissus mous, cancer du col et de l'utérus, } \\
\text { prostate, rabdomyosarcome, cancer bronchique à petites cellules, } \\
\text { médulloblastome, cancer de l'ovaire }\end{array}$ & Néoplasies & {$[16-20]$} \\
\hline $\begin{array}{l}\text { Leucémies T, lymphome B post-transplantation EBV induit, leucémie } \\
\text { lymphoïde chronique, leucémies aiguës myéloïdes et lymphoïdes }\end{array}$ & Hémopathies & [21] \\
\hline Hamartomes, angiomyolipomes & Phacomatoses & {$[9,22,23]$} \\
\hline
\end{tabular}

Tableau I. Types cellulaires cibles de l'inhibition de mTOR et applications cliniques avérées ou potentielles. 


\section{Applications en médecine cardiovasculaire}

À six mois après la pose d'une endoprothèse coronaire, $20 \%$ à $40 \%$ des patients développent une resténose dite intra-stent. L'hyperplasie intimale des cellules musculaires lisses est responsable de ce phénomène. La technique de l'enrobage (coating) de l'endoprothèse avec une molécule pharmacologiquement active s'est donc développée pour apporter in situ de fortes concentrations de produit et limiter ainsi l'hyperplasie. II a été démontré récemment que l'éradication quasi complète de la resténose par une endoprothèse enrobée de rapamycine était possible à six mois dans une population sélectionnée [15]. Par ailleurs, la Rapa a montré une certaine efficacité dans le ralentissement de la maladie athéromateuse dans un modèle de souris invalidée pour le gène de l'apolipoprotéine $\varepsilon$ (Tableau I).

\section{Applications en oncologie}

Le potentiel oncogénique des anomalies de signalisation des voies $\mathrm{PI} 3 \mathrm{k} / \mathrm{Akt} / \mathrm{mTOR}$ et PTEN/TSC/Rheb justifie l'importance de l'inhibition de mTOR en oncologie, et plusieurs études précliniques ont mis en évidence une efficacité des inhibiteurs de mTOR dans de très nombreux types de cancers [16].

Deux esters de la Rapa d'action analogue, l'évérolimus et le $\mathrm{CCl}-779$ ont ainsi été développés avec des propriétés pharmacocinétiques améliorées [17].

L'utilisation du CCl-779 dans les cancers du sein à un stade avancé est encourageante puisque son introduction après deux lignes de chimiothérapie a été suivie d'une efficacité clinique notable au prix d'une toxicité acceptable [18]. Le CCl-779 a également été étudié dans le cancer du rein à un stade avancé avec un taux de réponse global de $33 \%$ et une toxicité modérée [19]. Une étude de phase II récente a montré que le $\mathrm{CCl}$ 779 pouvait avoir une efficacité clinique et radiologique dans le traitement des glioblastomes en rechute [20]. Des résultats encourageants ont été obtenus avec le CCl-779 dans des études précliniques et chez des patients atteints de leucémies aiguës, myéloïdes et lymphoïdes de l'adulte [21] (Tableau I).

\section{Applications potentielles dans les phacomatoses et la polykystose rénale autosomique dominante}

Les phacomatoses, maladies au cours desquelles se développent des tumeurs bénignes (hamartomes) et des tumeurs malignes rénales, sont souvent liées à une activation de mTOR par l'intermédiaire d'une dysrégulation du complexe $\mathrm{TSC1} / \mathrm{TSC}$. Les mutations et la perte d'hétérozygotie des gènes TSC1 ou TSC2 sont responsables notamment de la sclérose tubéreuse de Bourneville. II a pu être démontré que l'inhibition de mTOR, dans des modèles murins de phacomatose, donnait des résultats prometteurs. Un essai clinique est en cours, évaluant l'intérêt de la Rapa comme traitement des angiomyolipomes [9, 22].

L'existence d'anomalies de prolifération des cellules épithéliales tubulaires rénales contribue à la kystogenèse observée au cours de la polykystose rénale auto- 
somique dominante (PKRAD). Dans un modèle de rat mimant la PKRAD humaine, le traitement à long terme par Rapa a permis de prévenir la kystogenèse et la perte de fonction rénale [23].

\section{Effets indésirables liés à l'utilisation des inhibiteurs de mTOR}

En transplantation rénale particulièrement, la notion de néphrotoxicité de la Rapa a émergé progressivement à la phase clinique de son développement. Sont actuellement bien caractérisés, des phénomènes de potentialisation de la néphrotoxicité de la CsA et de prolongation du délai de reprise de fonction rénale après greffe qui surviennent dans $10 \%$ à $50 \%$ des cas selon les études et nécessitent souvent une réduction de posologie, voire un arrêt du traitement $[12,25]$. Plus rarement, ont été décrits des microangiopathies thrombotiques (inhibition de la cicatrisation endothéliale) et la survenue de protéinuries [26, 27]. Le mécanisme des protéinuries sous Rapa est mal compris et pourrait impliquer à la fois un effet hémodynamique, par augmentation de la pression de perfusion glomérulaire, lié à la levée de la vasoconstriction induite à l'arrêt des inhibiteurs de la calcineurine (la Rapa étant alors utilisée comme alternative aux inhibiteurs de la

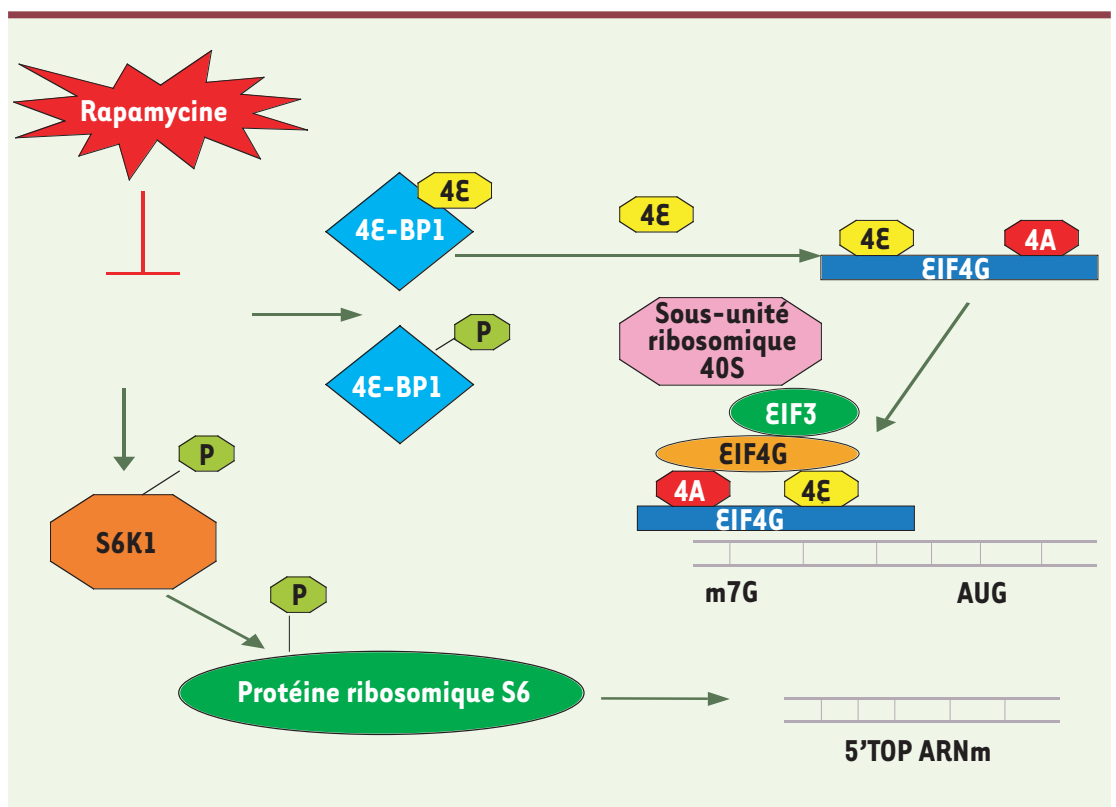

Figure 3. mTOR régule l'activité traductionnelle. L'assemblage du complexe ribosomique d'initiation de la traduction en $5^{\prime}$ des ARNm porteurs d'une coiffe 7-méthyl-guanosine $\left(\mathrm{m}^{7} \mathrm{G}\right)$ est représenté dans la partie supérieure du schéma. mTOR phosphoryle $4 \mathcal{E}-\mathrm{BPI}$ qui se dissocie alors de elF4E. Ce dernier est disponible pour former le complexe elF4F formé de elF4A, elF4G et elF4E. elF4F se lie à elF3 qui, à son tour, recrute la sous-unité ribosomique $40 S$ et initie alors la traduction. MTOR phosphoryle et active la S6K1, qui, à son tour, phosphoryle la protéine ribosomique $\$ 6$. Des données récentes indiquent que le contrôle de la traduction des 5'TOP ARNm n'est pas dépendant de la S6Kl ni de la phosphorylation de S6. calcineurine) et à une dysrégulation de certaines fonctions podocytaires. Il est à noter que, dans un modèle de glomérulonéphrite extramembraneuse avec inflammation interstitielle induite par la protéinurie, la Rapa réduisait le débit de protéinurie par son action anti-inflammatoire [28].

La rapamycine est responsable de retards de cicatrisation et de lymphocèles directement imputables à son effet sur la cicatrisation des tissus cutanés et lymphatiques. La Rapa est également responsable d'effets secondaires dermatologiques à la fois cutanés (acné, hydrosadénites, folliculites, lymphocèle), muqueux (aphtes, épistaxis, gingivites) et unguéaux [29].

D'autres effets secondaires ne semblent pas imputables aux propriétés antiprolifératives de la Rapa. À titre d'exemple, I'utilisation de Rapa est associée à la survenue de dyslipidémies mixtes chez $40 \%$ à $80 \%$ des patients sensibles aux statines, souvent réversibles à l'arrêt du traitements et pour lesquelles il n'a pu être mis en évidence de majoration du risque cardiovasculaire [30].

\section{Conclusions}

La découverte de la Rapa a permis de découvrir le rôle fondamental de la protéine kinase mTOR et de ses effecteurs S6K et $4 \varepsilon-B P I$ dans la régulation de la croissance cellulaire. L'inhibition de mTOR par la Rapa ou ses analogues est responsable d'une inhibition de la croissance d'un grand nombre de types cellulaires.

Les caractéristiques immunosuppressives de l'inhibition de mTOR couplées aux effets antitumoraux et les données récentes dans des modèles murins concernant la progression de l'athérosclérose donnent à ces molécules une place privilégiée en transplantations d'organe solide au cours desquelles la maladie athéromateuse et les cancers constituent les principales causes de morbidité et de mortalité à long terme.

Compte tenu de la place centrale de mTOR dans le contrôle de la croissance cellulaire, des applications en oncologie et en cardiologie ont été développées avec un grand succès. D'autres applications reposant sur les caractéristiques ubiquistes de mTOR verront certainement le jour dans les années à venir. $\diamond$

\section{SUMMARY}

MTOR inhibitors:

pleiotropic antiproliferative drugs

Studies of rapamycin properties in yeast led to the discovery of TOR (target of rapamycin) and its mammalian analogue, mTOR. mTOR is a central regulator 
of cell growth and proliferation in response to environmental stimuli such as growth factors and nutrients. mTOR regulates several pathways, particularly translation process by controling the activity of two proteins in response to a broad range of mitogenic stimuli, S6Kl and $4 \varepsilon-B P 1$. Inhibition of cell growth by rapamycine and analogues have been demonstrated in numerous cell types, explaining the broad development of these drugs in clinical practice. Rapamycine is a potent immunosuppressive drug used in solid organ transplantation for the prevention of allograft rejection. In oncology, mTOR inhibitors are currently evaluated in several types of cancers. They are now widely used for coating stents to reduce post-stenting restenosis after coronary angioplasty. Finally, rapamycine is now evaluated in various diseases characterized by cell growth disorders such as phacomatosis and autosomal dominant polycystic kidney disease. $\diamond$

\section{RÉFÉRENCES}

1. Heitman J, Movva NR, Hall MN, et al. Targets for cell cycle arrest by the immunosuppressant rapamycin in yeast. Science $1991 ; 253: 905-9$.

2. Sabers CJ, Martin MM, Brunn GJ, et al. Isolation of a protein target of the FKBP12-rapamycin complex in mammalian cells. J Biol Chem $1995 ; 270: 815-223$.

3. Tee AR, Blenis J. mTOR, translational control and human disease. Semin Cell Dev Biol 2005 ; $16: 29-37$.

4. Loewith $R$, Jacinto $\varepsilon$, Wullschleger $S$, et al. Two TOR complexes, only one of which is rapamycin sensitive, have distinct roles in cell growth. Mol Cell $2002 ; 10: 457-68.6$.

5. Wullschleger S, Loewith R, Hall MN. TOR signaling in growth and metabolism. Cell 2006; $124: 471-84$.

6. Schmelzle T, Tobias MN. MTOR : a central controller of cell growth. Cell $2000 ; 103: 253-62$.

7. Gangloff YG, Mueller M, Dann SG, et al. Disruption of the mouse mTOR gene leads to early postimplantation lethality and prohibits embryonic stem cell development. Mol Cell Biol $2004 ; 21: 9508-16$.

8. Ruvinsky I, Sharon N, Lerer T, et al. Meyuhas ribosomal protein $\mathrm{S} 6$ phosphorylation is a determinant of cell size and glucose homeostasis. Genes Dev $2005 ; 19: 2199-211$.

9. Beretta L, Gingras AC, Svitkin YV, et al. Rapamycin blocks the phosphorylation of $4 \varepsilon-B P I$ and inhibits cap-dependent initiation of translation. EMBO J 1996; $15: 658-6413$.

10. Harrington LS, Finlay GM, Lamb RF et al. Restraining PI3K : motor signalling goes back to the membrane. Trends Biochem Sci $2005 ; 30: 35-4$.

11. Inoki K, Corradetti MN, Guan KL, et al. Dysregulation of the TSC mTOR pathway in human disease. Nat Genet $2005 ; 37: 19-25.16$.

12. Kahan $B D$ for the rapamune US study group. Efficacy of sirolimus compared with azathioprine for the reduction of acute renal allograft rejection : a randomised multicentre study. Lancet $2000 ; 356: 194-20$.

13. Cahill BC, Somerville KT, Crompton JA, et al. Early experience with sirolimus in lung transplant recipients with chronic allograft rejection. J Heart Lung Transplant $2003 ; 22$ : 169-76.

14. Eisen HJ, Tuzcu EM, Dorent R, et al. RAD B253 Study Group. Everolimus for the prevention of allograft rejection and vasculopathy in cardiac-transplant recipients. $N$ Engl J Med 2003 ; $349: 847-58$.
15. Moses JW, Leon MB, Popma JJ, et al. Sirolimus-eluting stents versus standard stents in patients with stenosis in a native coronary artery. N Engl J Med 2003; 349 : 1315-23.

16. Bjornsti MA, Houghton PJ. The TOR pathway : a target for cancer therapy. Nat Rev Cancer $2004 ; 4: 335-41$.

17. Chan $\mathrm{S}$. Targeting the mammalian target of rapamycin (mTOR) : a new approach to treating cancer. BrJ Cancer $2004 ; 91$ : 1420-4.

18. Chan $S$, Scheulen $M E$, Johnston $S$, et al. Phase II study of temsirolimus (CCl-779) in heavily pretreated patients with locally advanced or metastatic breast cancer. J Clin Oncol 2005; 23 : 5314-49.

19. Atkins MB, Hidalgo M, Stadler WM, et al. Randomized phase II study of multiple dose levels of $\mathrm{CCl}-779$, a novel mammalian target of rapamycin kinase inhibitor, in patients with advanced refractory renal cell carcinoma. J Clin Oncol $2004 ; 22$ : 909-18.

20. Galanis $\varepsilon$, Buckner JC, Maurer MJ, et al. Phase II trial of Temsirolimus (CCl-779) in recurrent glioblastoma multiforme : a North central cancer treatment group study. Clin Oncol $2005 ; 10: 5294-9$.

21. Panwalkar A, Verstovsek S, Gilles FJ. Mammalian target of rapamycin inhibition as therapy for hematological malignancies. Cancer 2004 ; $100: 657-66$.

22. $\varepsilon$-Hashemite $\mathrm{N}$, Zhang $\mathrm{H}$, Henske FD. Mutation in TSC-2 and actication of mammalian target of rapamycine signaling pathway in renal angiolipoma. Lancet $2003 ; 361: 1346-9$.

23. Tao Y, Kim J, Schrier RW, et al. Rapamycine markedly slows disease progression in a rat model of polycystic kidney disease. J Am Soc Nephrol $2005 ; 16: 46-51$.

24. Podder H, Stepkowski SM, Napoli KL, et al. Pharmacokinetic interactions augment toxicities of sirolimus/cyclosporine combination. J Am Soc Nephrol 2001; 12 : 1059-66.

25. Pallet $N$, Thervet $\varepsilon$, Le Corre $D$, et al. SRL inhibits human epithelial cells proliferation: effect on cyclin D3 mRNA expression and stability. Kidney Int $2005 ; 67: 2422-30$.

26. Letavernier $\varepsilon$, Peraldi $M$, Pariente $A$, et al. Proteinuria following a switch from calcineurin inhibitors to sirolimus. Transplantation 2005; $80: 1198-207$.

27. Sartelet $H$, Toupance 0 , Lorenzato M, et al. Sirolimus-induced thrombotic microangiopathy is associated with decreased expression of vascular endothelial growth factor in kidney. Am J Transplant $2005 ; 5$ : 2441-5.

28. Bonegio RG, Fuhro R, Wang Z, et al. Rapamycin ameliorates proteinuriaassociated tubulointerstitial inflammation and fibrosis in experimental membranous nephropathy. J Am Soc Nephrol 2005 ; 7 : 2063-72.

29. Mahe $\varepsilon$, Morelon $\varepsilon$, Lechaton $S$, et al. Cutaneous adverse events in renal transplant recipients receiving sirolimus-based therapy. Transplantation. $2005 ; 27: 476-82$.

30. Chueh SC, Kahan B. Dyslipidemia in renal transplant recipients treated with a sirolimus and cyclosporine-based immunosuppressive regimen: incidence, risk factors, progression, and prognosis. Transplantation $2003 ; 27: 375-80$.
TIRÉS À PART

N. Pallet
Retrouvez chaque mois médecine/sciences sur France-Info dans la chronique « Info-Sciences 》 de Marie-Odile Monchicourt, du lundi au mercredi. france-info.com 